\title{
Duración de las internaciones psiquiátricas: una caracterización clínica y socio-demográfica
}

\author{
Damián Chebar ${ }^{1}$, Patricio Alba ${ }^{1}$, Héctor Alarcón ${ }^{1}$, Guillermo Fassi ${ }^{1}$, \\ Valeria Losanovsky Perel $^{1}$, Silvina Mazaira ${ }^{1}$, Diego J. Martino ${ }^{2}$
}

1. Unidad Internación de mujeres, Hospital de Emergencias Psiquiátricas "T. de Alvear", Buenos Aires, Argentina.

2. Doctor en Medicina, Consejo Nacional de Investigaciones Científicas y Técnicas (CONICET), Buenos Aires, Argentina.

Autor correspondiente: Damián Chebar, E-mail: damianchebar@gmail.com

\begin{abstract}
Resumen
Objetivo: Este trabajo busca determinar las variables clínicas, socio-demográficas, y terapéuticas asociadas a la duración de la internación en un Hospital de Emergencias Psiquiátricas de la Ciudad Autónoma de Buenos Aires (CABA), Argentina. Método: El presente es un estudio analítico, de tipo transversal, en el que se incluyeron consecutivamente 350 pacientes de entre 18 y 65 años, internadas desde junio de 2013 hasta diciembre de 2017 en la sala de internación de mujeres del Hospital de Emergencias Psiquiátricas "T. de Alvear". Al momento del alta se recabaron datos sociodemográficos, clínicos y sobre las condiciones de externación de todas las pacientes. Resultados: Las variables que predijeron la duración de la internación fueron: diagnóstico de psicosis, la utilización de litio y anticonvulsivantes, estar desocupada, menor autonomía económica, no haber constituido una familia y haber modificado el núcleo conviviente durante la internación. Conclusión: Las variables representativas de vulnerabilidad social y económica se asociaron con la utilización de camas de internación psiquiátrica. Son necesarias políticas públicas que permitan interrumpir la relación entre pobreza y patología mental.
\end{abstract}

Palabras claves: Internación psiquiátrica - Duración de la internación - Predictores - Vulnerabilidad social.

\section{LENGHT OF PSYCHIATRIC HOSPITALIZATIONS: A CLINICAL AND SOCIO-DEMOGRAPHIC CHARACTERIZATION}

\begin{abstract}
Aim: The aim of this study was to determine the clinical, socio-demographic, and therapeutic variables associated with the length of hospitalization in a psychiatric emergency hospital in Buenos Aires City. Method: The present retrospective analytical study included 350 consecutively admited patients aged 18-65, from June 2013 until December 2017 in a public psychiatric hospital in Buenos Aires City. Data collected included socio-demographic, clinical and discharge conditions. Results: Variables that predicted the length of hospitalization were: diagnosis of psychosis, the use of lithium and anticonvulsants, unemployment, no economic autonomy, not have formed a family and have modified the living support group during the hospitalization. Conclusion: The representative of social and economic vulnerability variables were associated with the utilization of psychiatric inpatient beds. Public policies are requested to interrupt the relationship between poverty and mental pathology.
\end{abstract}

Keywords: Psychiatric hospitalization - Lenght of stay - Predictors - Social vulnerability. 


\section{Introducción}

Las internaciones psiquiátricas han reducido sensiblemente su duración en las últimas décadas $(1,3)$. Este cambio puede ser explicado por varios motivos, como el desarrollo de los psicofármacos a partir de la década del '50, el cambio de paradigma hacia un abordaje comunitario, y la creciente preocupación de los pagadores por los costos en salud.

De todos modos, muchos autores critican la actual tendencia a llevar adelante internaciones cortas, en la medida que solo abordan el problema de la "peligrosidad" y la resolución de la crisis mediante el abordaje psicofarmacológico, con una mirada centrada en lo biológico y que deja de lado aspectos psicosociales fundamentales (1). Otros autores asocian esta progresiva disminución en los tiempos de internación con un aumento en las tasas de suicidio y actos criminales por parte de pacientes con patologías mentales $(2,3)$.

A pesar de los avances realizados en términos de desinstitucionalización y reinserción de los usuarios, en el Reino Unido todavía casi el 10\% de los pacientes internados permanece más de 90 días y el 1\% más de un año (4). En Francia, estas últimos ascienden al 2,5\% (5). Las conceptualizaciones sobre la definición de internación prolongada distan de ser homogéneas (2). Diferentes trabajos utilizan periodos que van desde los 28 a los 360 días (1,3-6). Otros autores utilizan una definición relativa, considerando como tal a aquellas incluidas en el percentilo 97,5 de los días de internación (7). Esta situación dificulta notablemente las comparaciones entre estudios y definir un tiempo "adecuado" de internación.

Existen varios factores de riesgo, tanto del paciente como de los sistemas de salud, asociados a las internaciones prolongadas, aunque estas evidencias han sido calificadas como de baja calidad (2). Entre los factores asociados a los pacientes se ha descrito el tener un diagnóstico de psicosis (3-5,8-10), severidad de la enfermedad $(3,4$, $5)$ antecedentes de conductas violentas (4), género masculino $(5,9)$, género femenino (8), estar desempleado $(9$, $11)$, ser soltero $(3,8)$, necesidad de brindarle alojamiento $(4,9)$, vivir en grandes centros urbanos (5) y pertenecer a minorías étnicas $(4,8,9)$. Paradójicamente, la suicidalidad al ingreso se asoció a estadías más breves (4). Respecto a los factores del sistema de salud, la preferencia del jefe de servicio en relación a la duración de la internación (3), la menor cantidad de camas en el sector (5), y la realización de cambios en el plan psicofarmacológico (3) también se asociaron a internaciones prolongadas.

Nuestro país se encuentra en una situación de transición donde contamos con una legislación de fuerte impronta comunitaria, pero en un contexto de escasez de dispositivos intermedios entre la internación y el tratamiento ambulatorio. En esta coyuntura la búsqueda de los factores asociados a la duración de la internación adquiere relevancia para poder realizar un uso racional y eficiente de los recursos existentes. Sin embargo, en la actualidad no contamos en nuestro medio con datos res- pecto de los determinantes del tiempo de hospitalización. Por lo tanto, el objetivo del presente trabajo fue evaluar las variables sociodemográficas, clínicas, y terapéuticas asociadas a la duración de la internación.

\section{Material y métodos}

El presente es un estudio analítico, de tipo transversal, en el que se incluyeron consecutivamente pacientes de entre 18 y 65 años externadas entre el 2013 y el 2017 en la sala de internación de mujeres del Hospital de Emergencias Psiquiátricas "T. de Alvear" de la Ciudad Autónoma de Buenos Aires (CABA), Argentina.

Las pacientes ingresan a la sala únicamente por derivación desde la guardia externa del hospital y tiene una capacidad de 30 camas. Se trata de un dispositivo para pacientes subagudos con la presencia permanente de dos enfermeros. El criterio de derivación a la sala suele ser la imposibilidad de un alta pronta por guardia en base a la complejidad clínica, social y familiar. Para cada paciente se relevaron variables sociodemográficas, clínicas y terapéuticas. Estos datos fueron volcados en un formulario, y completados por cada psiquiatra tratante al momento del alta.

Los diagnósticos fueron realizados por médicos del servicio con más de 10 años de experiencia clínica, según los criterios del CIE-10. Los datos relacionados con la situación habitacional y laboral fueron organizados con criterios similares a los utilizados en el último censo nacional, aunque con algunas modificaciones realizadas por los autores vinculadas a la experiencia de trabajo. En aquellos casos que presentaron más de una internación en el hospital durante el período de estudio, sólo se consignaron los datos de la última con la finalidad de que todas las observaciones puedan ser consideradas independientes. Todas las pacientes (o sus representantes legales) firmaron un consentimiento informado al momento del alta, previo al relevamiento de los datos del estudio y luego de haber sido adecuadamente informadas acerca del alcance del mismo.

Respecto al análisis estadístico, la normalidad de las variables se evaluó mediante métodos analíticos (Kolmogorov-Smirnov). Dada la distribución asimétrica de la mayoría de las variables, la comparación entre grupos de variables continuas se realizó utilizando el test de Mann-Whitney y Kruskal-Wallis. Incluso en el caso de variables no paramétricas, se utilizaron la media y desvío estándar como medidas descriptivas para facilitar la comprensión. Las variables cualitativas se analizaron a través del test de chi-cuadrado con corrección de continuidad. La asociación entre variables se evaluó a través de los coeficientes de correlación de Spearman.

\section{Resultados}

Durante el período de estudio se incluyeron 350 pacientes con una edad media de 36.59 (Desvío Estándar=11.67) años y 11.26 (2.82) años promedio de educación. Las mismas estuvieron internadas durante 97.37 
(67.89) días, predominando las internaciones involuntarias $(67.9 \%)$ sobre las voluntarias $(32.1 \%)$. Las características sociodemográficas se muestran en la Tabla 1.

Tabla 1. Características sociodemográficas de las pacientes al momento del ingreso

\begin{tabular}{|c|c|}
\hline Variable & Porcentaje válido \\
\hline Nacionalidad argentina & 90.8 \\
\hline \multicolumn{2}{|l|}{ Estado Civil } \\
\hline Soltera & 57.0 \\
\hline Casada/Conviviente & 18.7 \\
\hline Separada & 20.8 \\
\hline Viuda & 3.5 \\
\hline \multicolumn{2}{|l|}{ Lugar de vivienda } \\
\hline CABA & 45.0 \\
\hline Gran Buenos Aires & 52.1 \\
\hline Otros & 2.9 \\
\hline \multicolumn{2}{|l|}{ Tipo de Vivienda } \\
\hline Material & 90.7 \\
\hline Casilla precaria & 1.2 \\
\hline Pensión/Institución & 7.2 \\
\hline Situación de calle & 0.9 \\
\hline \multicolumn{2}{|l|}{ Situación de Vivienda } \\
\hline Propia & 35.5 \\
\hline Alquilada & 19.9 \\
\hline Familiar/Prestada & 39.0 \\
\hline Otras & 5.6 \\
\hline \multicolumn{2}{|l|}{ Trabajo } \\
\hline Trabajo formal / Tiempo completo & 12.0 \\
\hline Trabajo precario / A tiempo parcial & 14.6 \\
\hline No busca trabajo (ama de casa) & 29.8 \\
\hline Desocupada & 43.6 \\
\hline \multicolumn{2}{|l|}{ Autonomía Económica } \\
\hline Autónoma / No recibe ayuda & 16.6 \\
\hline Ama de casa & 10.8 \\
\hline Recibe ayuda de pareja o familia & 48.3 \\
\hline Recibe pensión o plan social & 21.5 \\
\hline Otros & 2.9 \\
\hline \multicolumn{2}{|l|}{ Tipo de cobertura de salud } \\
\hline Obra social / Prepaga & 22.8 \\
\hline Ninguna & 72.2 \\
\hline Certificado de discapacidad & 28.7 \\
\hline Pensión por invalidez & 22.8 \\
\hline
\end{tabular}

El $66 \%$ de la muestra $(n=231)$ tenían antecedentes de internaciones psiquiátricas previas (media=2.12, $\mathrm{DE}=2.74$ ). Los diagnósticos principales de las pacientes internadas fueron esquizofrenia y otros trastornos psicóticos (27.7\%), depresión unipolar (12.6\%), trastorno bipolar -incluyendo tanto depresión como manía(27.1\%), y trastornos de la personalidad (mayoritariamente de tipo limítrofes) (22.9\%). El resto de las pacientes $(9.7 \%)$ tuvieron diagnósticos menos frecuentes como trastornos de ansiedad, demencias, o discapacidad intelectual, entre otros. El $18.7 \%$ de la muestra presentó comorbilidad con abuso de sustancias (excluyendo el alcohol), mientras que el $15.0 \%$ presentó comorbilidad con abuso de alcohol.

Al momento del ingreso, el 13.7\% de las pacientes vivían solas, el 33.1\% con sus padres, el 20.9\% en pareja con o sin hijos, el $17.7 \%$ con hijos (con o sin sus propios padres), mientras que el restante $14.6 \%$ vivía con otros familiares, amigos/as, en instituciones, etc. A lo largo de la internación el $28.4 \%$ de la muestra cambió su situación habitacional, siendo externado a un lugar de vivienda diferente al del ingreso. La externación en la mayoría de los casos fue por alta médica (93.1\%), mientras que sólo en el $4.8 \%$ de los casos fue por abandono y en el $2.1 \%$ por traslados a otra institución.

\section{Factores asociados a la duración de la internación}

Como se mencionó más arriba, las pacientes incluidas estuvieron internadas un promedio de 97.37 (67.89) días. Las internaciones fueron más prolongadas entre las pacientes con antecedentes de internaciones previas que entre las que no las tenían $(103.86 \pm 71.34$ vs. 86.78 $\pm 58.93 ; \mathrm{Z}=-2.14, \mathrm{p}=0.033$ ), aunque no mostraron relación con el tiempo transcurrido desde la última internación.

Entre las variables sociodemográficas analizadas, la duración de la internación se asoció con el tipo de vivienda (Kruscal Wallis X2=5.68; $\mathrm{gl}=3 ; \mathrm{p}=0.007$ ), siendo menor entre las pacientes que tenían una de material (93.44 \pm 64.64 días) que entre aquellas que vivían en pensiones/instituciones (129.32 \pm 67.87 ; Mann-Whitney $\mathrm{Z}=-3.05 ; \mathrm{p}=0.002)$ o en situación de calle (212.00 \pm 166.00 ; $\mathrm{Z}=-1.82 ; \mathrm{p}=0.038)$. Se encontró una asociación en relación a la situación de vivienda (Kruscal Wallis X2=9.24; $\mathrm{gl}=3 ; \mathrm{p}=0.026$ ), siendo mayor el tiempo de internación en aquellas pacientes que no tenían vivienda propia, alquilada, o familiar (133.68 \pm 73.44 días). Asimismo, tanto aquellas pacientes con trabajo a tiempo completo o tiempo parcial tuvieron internaciones más breves (68.19 \pm 52.74 y $68.84 \pm 30.02$ días respectivamente) que aquellas desocupadas (108.75 $\pm 71.64 ; Z=-4.30, p<0.001$ y $Z=-3.70, p<0.001$ respectivamente) o que no buscaban trabajo (106.88 $\pm 74.25 ; Z=-3.88, p<0.001$ y $Z=-3.41$, $\mathrm{p}=0.001$ respectivamente). La autonomía económica también se asoció con los días de internación (Kruscal Wallis $\mathrm{X} 2=17.87 ; \mathrm{gl}=4 ; \mathrm{p}=0.001$ ), con hospitalizaciones más prolongadas entre las pacientes que recibían pensiones/planes sociales $(119.30 \pm 89.26)$. No hubo dife- 
rencias en los días de internación respecto del tipo de cobertura de salud, aunque las pacientes con certificado de discapacidad tuvieron internaciones más prolongadas que aquellas que no lo tenían (126.28 \pm 88.80 vs. 85.88 $\pm 53.43 ; \mathrm{Z}=-4.27, \mathrm{p}<0.001$ ).

En relación a las variables clínicas, los diagnósticos mostraron tener un efecto sobre el tiempo de internación (Kruscal Wallis X2=18.21; gl=4; p=0.001). Los análisis post-hoc mostraron que las pacientes con esquizofrenia y otros trastornos psicóticos tuvieron internaciones más largas (119.69 \pm 79.51 días) que las pacientes con depresión unipolar (86.39 \pm 53.89 días; $Z=-2.63$, $\mathrm{p}=0.009)$, trastorno bipolar $(91.19 \pm 56.08$ días; $\mathrm{Z}=-2.69$, $\mathrm{p}=0.007), \mathrm{y}$ trastornos de personalidad $(80.13 \pm 53.74$ días; $\mathrm{Z}=-3.80, \mathrm{p}<0.001)$. La comorbilidad con abuso de alcohol o sustancias no se asoció con incrementos en los tiempos de internación en la muestra entera ni tampoco cuando se evaluaron por separado en los diagnósticos en los que eran más prevalentes (trastornos de personalidad y trastorno bipolar). Entre los psicofármacos utilizados tanto el agregado $(\mathrm{Z}=-2.55, \mathrm{p}=0.011)$ como el retiro $(\mathrm{Z}=-$ 2.44, $\mathrm{p}=0.015$ ) de drogas antiepilépticas a lo largo de la internación se asociaron con incrementos en la estadía hospitalaria respecto de aquellos pacientes que no recibieron cambios en este grupo de drogas (ya sea porque nunca las tomaron o porque lo hicieron desde el ingreso hasta el egreso). Asimismo, el cambio a lo largo de la internación en la utilización del litio (el cual se debe fundamentalmente al agregado), se asoció a un incremento en los tiempos de internación $(\mathrm{Z}=-2.00 ; \mathrm{p}=0.046)$. Por el contrario, los cambios en los antipsicóticos típicos o atípicos, y antidepresivos no se asociaron a diferencias en la duración de la internación.

El núcleo conviviente también mostró un efecto en la duración de la internación (Kruscal Wallis X2=14.57; $\mathrm{gl}=4 ; \mathrm{p}=0.006$ ). Aquellas pacientes que vivían solas tuvieron internaciones más prolongadas ( $114.40 \pm 80.20$ días) que las que vivían en pareja $(80.78 \pm 58.62$ días; $Z=-2.82$, $\mathrm{p}=0.005)$ o con sus hijos $(83.77 \pm 50.79$ días; $\mathrm{Z}=-2.29$, $\mathrm{p}=0.022$ ). Aquellas pacientes que vivían con sus padres al momento del ingreso tuvieron internaciones de duración intermedia entre los otros grupos (105.30 \pm 69.92 días), las cuales no difirieron estadísticamente respecto de las que vivían solas $(\mathrm{Z}=-0.54, \mathrm{p}=0.59)$, pero fueron superiores a las de aquellas que vivían con parejas $(Z=-$ 3.02, $\mathrm{p}=0.003)$ o hijos $(\mathrm{Z}=-2.56, \mathrm{p}=0.010)$. Asimismo, el hecho de que se hayan realizado cambios en el núcleo conviviente durante la internación también prolongó la duración de la misma (117.94 \pm 75.23 vs. $89.43 \pm 62.56$; $\mathrm{Z}=-3.93, \mathrm{p}<0.001)$. Finalmente, las pacientes que fueron externadas por alta médica tuvieron una internación de duración intermedia $(98.19 \pm 64.89)$ entre aquellas que abandonaron el tratamiento $(52.07 \pm 39.44 ; \mathrm{Z}=-3.57$, $\mathrm{p}<0.001)$ y las que resultaron trasladadas a otra institución (201.29 $\pm 123.06 ; \mathrm{Z}=-2.39, \mathrm{p}=0.017)$.

Con el objetivo de valorar cuáles de todas estas variables podían ser predictoras independientes, se realizó un análisis multivariado de regresión lineal con la duración de la internación como variable dependiente. El modelo final que se muestra en la Tabla 2 permitió explicar más del 25\% de la duración de la internación (R2 corregido:0.264; $\mathrm{F}=13.78 ; \mathrm{p}<0.001)$.

Tabla 2. Predictores independientes de la duración de la internación en el modelo final de regresión lineal

\begin{tabular}{l|c|c|c}
\hline Modelo & Coeficientes $\beta$ & $\mathbf{t}$ & $\mathbf{p}$-valor \\
\hline Constante & 25.18 & 2.17 & 0.007 \\
\hline $\begin{array}{l}\text { Desocupada/no buscando } \\
\text { trabajo }\end{array}$ & 31.72 & 3.57 & $<0.001$ \\
\hline $\begin{array}{l}\text { Menor autonomía } \\
\text { económica }\end{array}$ & 27.23 & 2.92 & 0.004 \\
\hline $\begin{array}{l}\text { Sin familia constituida } \\
\text { solteras/viviendo con } \\
\text { padres) }\end{array}$ & 26.02 & 3.43 & 0.001 \\
\hline $\begin{array}{l}\text { Cambio en núcleo } \\
\text { conviviente }\end{array}$ & 19.63 & 2.33 & 0.021 \\
\hline $\begin{array}{l}\text { Esquizofrenia y otros } \\
\text { trastornos psicóticos }\end{array}$ & 31.35 & 3.63 & 0.001 \\
\hline $\begin{array}{l}\text { Cambio drogas } \\
\text { antiepilépticas }\end{array}$ & 42.51 & 4.82 & $<0.001$ \\
\hline Cambio litio & 21.70 & 2.25 & 0.025 \\
\hline
\end{tabular}

\section{Discusión}

La mayor parte de las pacientes incluidas tenían antecedentes de internaciones previas y mostraban una distribución homogénea entre trastornos psicóticos, afectivos, y de personalidad. Los datos de comorbilidad con abuso de alcohol y sustancias superaron el $25 \%$ en algunos diagnósticos lo cual también es acorde con datos previos (12-14). Es de destacar la situación de vulnerabilidad social de las mujeres incluidas en el estudio, considerando que, con una edad media de 36 años, el $77,8 \%$ se encuentran solteras o separadas, sólo el 35\% tiene una vivienda propia y menos del 20\% tienen trabajo formal y autonomía económica. La falta de autonomía económica y la escasa proporción de usuarias que contaban con un trabajo formal, ya sea en relación de dependencia o por cuenta propia en los dos meses previos, se encuentran en línea con los datos de la bibliografía. Se calcula que en el mundo entre el $60 \mathrm{y}$ el $85 \%$ de las personas con trastornos mentales severos se encuentran desocupados, incluyendo las cifras de los países desarrollados y con políticas activas de inclusión $(15,16,17)$.

En la muestra estudiada el $28,4 \%$ requirió para su externación modificar el entorno de residencia del paciente. En otras palabras 1 de cada 4 pacientes no volvieron al entorno en el que convivían antes de su internación. En la práctica, este cambio puede requerirse en diferentes situaciones: por la negativa de la paciente a regresar con el núcleo conviviente, si la familia no resulta continente o se niega a recibir nuevamente a la paciente, o por la falta de condiciones mínimas de habitabilidad (por ej. situación de calle). 
La duración promedio de la internación fue de 97.37 días, lo que implica tiempos de internación considerablemente superiores a la mayoría de los estudios (9, 18-21). Es probable que, al menos en parte, estos períodos de internación prolongados guarden relación con la situación de vulnerabilidad social de las pacientes evaluadas. De hecho, y tal como se ha observado en estudios previos $(5,9)$, la falta de trabajo y autonomía económica se asociaron a tiempos más prolongados de internación. La demora en obtener recursos habitacionales que permitan la externación (hogares, casas de medio camino, centros de día, etc.) es un factor que impacta definitivamente en los tiempos de internación, si bien no está actualmente cuantificado. En relación con esto, el cambio en el núcleo conviviente de las pacientes fue un predictor de mayor duración de la internación.

En la sala de internación se realiza un abordaje interdisciplinario en donde las pacientes cuentan con tratamiento psiquiátrico, psicoterapia individual y familiar, terapia ocupacional y abordaje de trabajo social. El impacto de este abordaje interdisciplinario en los tiempos de internación aún no ha sido estudiado.

En conjunto, muchos de estos resultados vuelven a poner de manifiesto la estrecha asociación entre pobreza y enfermedad mental. Al respecto existen diversas teorías que en sus extremos se definen como la hipótesis de la causa social y la hipótesis de la selección social $(22,23)$. La primera adjudica a las carencias y al estrés propio de las situaciones de pobreza un factor causal en la aparición de las enfermedades mentales. La teoría de la selección social propone que la propia patología le generaría desventajas al momento de competir con personas sanas. Sin pretender extendernos en este debate, los resultados de este trabajo ponen de manifiesto la necesidad de establecer estrategias que apunten a interrumpir el círculo pobreza-enfermedad mental. Como este trabajo estudia una muestra de mujeres internadas en una institución psiquiátrica, no podemos dejar de mencionar que se trata de una población que además sufre las desigualdades de género. Por tal motivo, entre las acciones necesarias para mejorar las condiciones de nuestras pacientes, se requiere un abordaje integral con perspectiva de género para lograr su empoderamiento.

También se observó que las pacientes con diagnóstico de esquizofrenia permanecieron internadas más tiempo que aquellas con trastornos afectivos o de la personalidad. El diagnóstico de psicosis ya ha sido asociado a la prolongación de la internación en múltiples estudios (3-5, 8-10). La falta de asociación con el consumo de alcohol y sustancias en este estudio podría deberse a que se utilizaron los criterios del CIE-10 para Trastorno por abuso de sustancias, no quedando representado el uso excesivo de estas sustancias en una población vulnerable.

Los cambios en el empleo de drogas antiepilépticas y litio durante la internación (que consistieron fundamentalmente en el agregado de estas drogas) también se asociaron a una mayor duración de la internación. Es probable que esta asociación se deba, al menos en parte, a que son fármacos que en muchos casos deben titularse lentamente (ej. lamotrigina) y que requieren dosajes plasmáticos para su ajuste (ej. litio, valproato, o carbamazepina). Por otra parte, en ciertos cuadros clínicos (como el trastorno depresivo mayor o los trastornos de personalidad) estos fármacos se emplean en casos refractarios a otros tratamientos, lo cual también podría contribuir a explicar la asociación encontrada.

La duración media prolongada también podría asociarse con un sesgo de selección de los pacientes que pasan a la sala de internación. Aquellas pacientes con posibilidades de un alta rápida suelen externarse en forma directa desde la guardia.

Un aspecto no evaluado en el presente trabajo es la influencia de las características de los equipos tratantes y la modalidad de abordaje en los tiempos de internación. En este sentido sería importante poder comparar estos datos con otros centros de características similares.

\section{Conclusiones}

En el presente trabajo se brinda una caracterización de la población internada en un hospital de emergencias psiquiátricas de la CABA donde se ponen de manifiesto los factores vinculados a la duración de las internaciones.

Es importante destacar la influencia de las características socioeconómicas de las pacientes y sus entornos en el uso de los dispositivos de internación y remarcar la necesidad de desarrollar programas adecuados que promuevan la integración social. En este sentido, se tornan imprescindibles políticas públicas que interrumpan el circulo pobreza-enfermedad mental, y que brinden sostén a las pacientes y sus familias.

\section{Declaración de conflictos de intereses}

Los autores declaran no presentar conflictos de intereses en relación al presente artículo. 


\section{Referencias bibliográficas}

1. Glick ID, Sharfstein SS, Schwartz HI. Inpatient psychiatric care in the 21st century: the need for reform. Psychiatr Serv. $2011 \mathrm{Feb} ; 62(2): 206-9$.

2. Capdevielle D, Ritchie K. The long and the short of it: are shorter periods of hospitalisation beneficial? Br J Psychiatry. 2008 Mar;192(3):164-5

3. Capdevielle D, Norton J, Jaussent I, Prudhomme C, Munro J, Gelly F, Boulenger JP, Ritchie K. Extended duration of hospitalization in first episode psychosis: an evaluation of its clinical justification. Psychiatry Res. 2013 Sep 30;209(2):160-6.

4. Tulloch AD, Fearon P, David AS. The determinants and outcomes of long-stay psychiatric admissions: a case-control study. Soc Psychiatry Psychiatr Epidemiol. 2008 Jul;43(7):56974.

5. Plancke L, Amariei A. Long-term psychiatric hospitalizations. Rev Epidemiol Sante Publique. 2017 Feb;65(1):9-16.

6. Babalola O1, Gormez V, Alwan NA, Johnstone P, Sampson S. Length of hospitalization for people with severe mental illness. Cochrane Database Syst Rev. 2014 Jan 30;(1):CD000384

7. Brown TM1, Miller HL, Ekstrom D, Evans DL, Golden RN. Characteristics of long-stay patients on the psychiatric service of a university hospital. Hosp Community Psychiatry 1991 Jul;42(7):743-5.

8. Tulloch AD, Fearon P, David AS. Length of stay of general psychiatric inpatients in the United States: systematic review. Adm Policy Ment Health. 2011 May;38(3):155-68.

9. Newman L, Harris V, Evans LJ, Beck A. Factors Associated with Length of Stay in Psychiatric Inpatient Services in London, UK. Psychiatr Q. 2018 Mar;89(1):33-43.

10. Hallak JE, Crippa JA, Vansan G, Zuardi AW. Diagnostic profile of inpatients as a determinant of length of stay in a general hospital psychiatric unit. Braz J Med Biol Res. 2003 Sep;36(9):1233-40.

11. Johnstone P, Zolese G. Systematic review of the effectiveness of planned short hospital stays for mental health care. BMJ. 1999 May 22;318(7195):1387-90.

12. Machado V, Leonidas C, Santos MA, Santos MA. Psychiatric readmission: an integrative review of the literature. Int Nurs Rev. 2012 Dec;59(4):447-57.
13. Donisi V, Tedeschi F, Wahlbeck K, Haaramo P, Amaddeo F. Pre-discharge factors predicting readmissions of psychiatric patients: a systematic review of the literature. BMC Psychiatry. 2016 Dec 16;16(1):449.

14. Jaramillo-Gonzalez E, Sanchez Pedraza, R y Herazo MI The frequency of rehospitalization and associated factors in Colombian psychiatric patients: a cohort study. BMC Psychiatry 2014, 14:161

15. Crowther R, Marshall M, Bond GR, Huxley P. Helping people with severe mental illness to obtain work: systematic review. BMJ. 2001 Jan 27;322(7280):204-8.

16. Kinoshita Y1, Furukawa TA, Kinoshita K, Honyashiki M, Omori IM, Marshall M, Bond GR, Huxley P, Amano N, Kingdon D. Supported employment for adults with severe mental illness. Cochrane Database Syst Rev. 2013 Sep 13;(9).

17. Marwaha S, Johnson S. Views and experiences of employment among people with psychosis: a qualitative descriptive study. Int J Soc Psychiatry. 2005 Dec;51(4):302-16.

18. Nemirovsky M, Guardo G, Rosenstein M, Agrest M, Tkach J, Montanelli R.. Epidemiological features of admitted patients to a psychiatric unit in a Prepaid Medical Organization in Buenos Aires City. Vertex, Revista Argentina de Psiquiatría. 2003 Dec-2004 Feb;14(54):292-8.

19. Serrani D, Galfetti G, Rodríguez C. Factors associated with length of stay and the risk of readmission in an acute psychiatric inpatient facility: A retrospective study. Vertex, Revista Argentina de Psiquiatría. 2017 Jul;28(134):260-270.

20. Thompson EE, Neighbors HW, Munday C, Trierweiler $S$, Length of stay, referral to aftercare, and hospitalization among psychiatric inpatients, Psychiatr Serv. 2003 Sep;54(9):1271-6.

21. Figueroa R, Harman J, Engberg J: Use of claims data to examine the impact of length of inpatient psychiatric stay on readmission rate. Psychiatr Serv. 2004 May;55(5):560-5.

22. Ritsher JE, Warner V, Johnson JG, Dohrenwend BP. Inter-generational longitudinal study of social class and depression: a test of social causation and social selection models. $\mathrm{Br} \mathrm{J}$ Psychiatry Suppl. 2001 Apr;40:84-90.

23. Dohrenwend BP, Levav I, Shrout PE, Schwartz S, Naveh G, Link BG, Skodol AE, Stueve A. Socioeconomic status and psychiatric disorders: the causation-selection issue. Science. 1992 Feb 21;255(5047):946-52. 